\title{
Stabilization of Contention-Based CDMA Ranging Channel in Wireless Metropolitan Area Networks
}

\author{
Jeong-Jae Won ${ }^{1}$, Choong-Ho Cho ${ }^{2}$, Hyong-Woo Lee ${ }^{3}$, and Victor C.M. Leung ${ }^{1}$ \\ 1 Department of Electrical and Computer Engineering, \\ The University of British Columbia, \\ 2356 Main Mall, Vancouver, BC, Canada V6T 1Z4 \\ $\{$ wonjj,vleung\}@ece.ubc.ca \\ 2 Department of Computer and Information Science, Korea University \\ 3 Department of Electronics \& Information Engineering, Korea University \\ $\{$ chcho, hwlee\}@korea.ac.kr
}

\begin{abstract}
The current IEEE 802.16a and 802.16d standards of wireless metropolitan area networks specify a ranging channel in the OFDMA physical layer that employs a set of CDMA codes for ranging and bandwidth requests. The ranging channel is contention-based and inherently unstable. In this paper, we propose two stabilization algorithms to enable efficient utilization of the ranging channel at close to the theoretical throughput limit, and analyze their performance using a continuous time Markov chain M/M/1 model. We show how to estimate system parameters such as the number of backlogged users, arrival rate and the first exit time for the critical. Based on these parameters, we present two methods for channel stabilization, for the cases that the number of ranging codes per frame is fixed and adjusted dynamically, respectively. We then present simulation results to show that, by restricting the actual arrival rate or dynamically adjusting the number of ranging codes, the ranging channel can be stabilized under all traffic conditions.
\end{abstract}

Keywords: Wireless metropolitan area network (WMAN), OFDMA, CDMA, Ranging channel, Ranging code, System stability.

\section{Introduction}

The new IEEE 802.16a standard [1] and 802.16d draft standard [2] for wireless metropolitan area networks (802.16 WMANs) specify a protocol that among other things support real-time applications such as voice and video, provides broadband connectivity without requiring a direct line of sight between the subscriber station (SS) and the base station (BS), and support hundreds if not thousands of SSs from a single BS. A SS can be a fixed terminal or a mobile terminal (MT). The standard will help accelerating the introduction of broadband wireless equipment into the market, speeding up last-mile broadband deploy- 
ment worldwide by enabling service providers to increase system performance and reliability while reducing their equipment costs and investment risks [3].

In this paper, we focus on the contention-based ranging/bandwidth request channel in 802.16 WMANs, which employs a set of CDMA ranging codes over the OFDMA physical layer. We refer to this system as OFDMA-CDMA multichannel ALOHA (OC-ALOHA) since this system has properties similar to slotted ALOHA. Subsets of ranging codes are allocated in the uplink channel descriptor (UCD) for initial ranging, periodic ranging and bandwidth requests. In [1], [2], an SS transmits a ranging code chosen at random from the assigned subset of codes in one of the slots, again chosen at random from the assigned set of slots. If no other SS transmits the same code in the same slot, the code will be successfully received by the BS. Otherwise a collision occurs and all the transmissions involved are destroyed. The SSs involving in the collision back off and retransmit.

As the access procedure is similar to slotted ALOHA [4], [5] which is inherently unstable, a relatively large set of ranging codes and ranging slots (referred here as code slots) is usually allocated for ranging and bandwidth requests. This allocation for ranging channel may also be altered by various reasons such as the dynamic adjustments of the sizes of uplink sub-frames inserted in uplink map (UL-MAP). Although the performance of existing random access protocols such as GPRS in [4], [5], WLAN in [5], [7] and HIPERLAN in [8], [9] has been widely studied, analysis of the contention-based CDMA ranging channel in 802.16 WMANs is still an open problem. In our previous work [6], the performance of the ranging channel has been evaluated by a continuous time Markov chain (CTMC) M/M/1 model which is approximated by the discrete time Markov chain (DTMC) model, as the exact DTMC model can become intractable. This analytical model allows the unique characteristics of the ranging system to be studied in detail.

Based on the CTMC M/M/1 model presented in [6], we propose in this paper two novel stabilization algorithms that enable the efficient utilization of the contention-based CDMA ranging channel in 802.16 WMANs. We first present a performance evaluation using the CTMC M/M/1 model to show how system parameters such as the number of backlogged users, arrival rate and the first exit time (FET) for the critical state can be estimated and utilized for system stabilization. Then, we present two methods for system stabilization, for the cases that the number of ranging codes per frame is fixed and adjusted dynamically, respectively. In the former case, the rate of ranging and bandwidth code requests that can be transmitted in the next frame is established based on the estimated system parameters. In the latter case, the number of ranging codes per frame is dynamically adjusted based on the estimated system parameters. We further present simulation results to how that the proposed algorithms can always maintain the stability of the ranging channel even if its normalized throughput approaches the theoretical maximum value of $36 \%$ for the slotted ALOHA channel. 
This paper is organized as follows. In section 2, we review the structure of the ranging channel and the ranging procedure specified in [1], [2]. In section 3, we present the channel stabilization algorithms and analyze their performance in terms of estimated arrival rate, estimated critical state and the estimated number of backlogged users. In section 4 , we present the simulation results in comparison with the Pseudo-Bayesian algorithm presented in [4], [10]. In section 5 , we draw some conclusions.

\section{Contention-Based CDMA Ranging Subchannel}

The medium access control (MAC) specifications in [1], [2] define a single ranging channel over the uplink (which we refer as the OC-ALOHA ranging channel) composed of one or more pairs of adjacent subchannels, where the index of the numbered subchannel of each pair is given as shown in Fig. 1. The indices of the subchannel pairs that compose the ranging channel are specified in the uplink message. Transmissions may collide on this ranging channel. To effect a ranging transmission, a SS randomly chooses one CDMA ranging code from among the appropriate sets specified for initial ranging, periodic ranging and bandwidth request.

The initial ranging transmission is sent by any SS that wants to synchronize to the system channel for the first time. An initial ranging transmission is performed during consecutive OFDMA symbols. The same CDMA ranging code is transmitted on the ranging channel during each symbol, with no phase discontinuity between two adjacent symbols. Periodic ranging transmissions are sent periodically to adjust system parameters such as timing and power level. SSs also send bandwidth request transmissions to request uplink channel allocations from the BS. These latter transmissions shall be sent only by the SSs that have already synchronized to the system. To perform either a periodic ranging or bandwidth request transmission, the SS shall modulate one CDMA ranging code on the ranging channel for a period of one OFDMA symbol. Ranging channel is dynamically allocated by the BS MAC and indicated in the UL-MAP message.

The procedures of ranging request are described in Fig. 2. The SS, after acquiring downlink synchronization and uplink transmission parameters, randomly

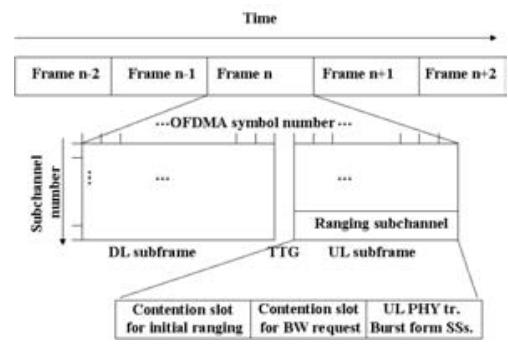

Fig. 1. The structure of OFDMA MAC frame 


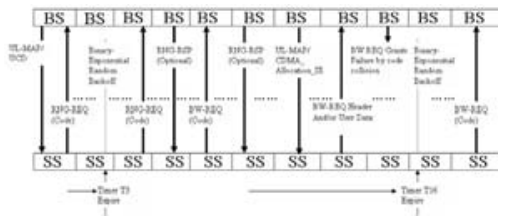

Fig. 2. The procedure of ranging code requests

chooses (a) a ranging slot (with the use of a truncated binary exponential backoff algorithm to avoid possible re-collisions) as the time to perform the ranging, and (b) a ranging code (from the subset of CDMA codes allocated for initial ranging), and sends it to the $\mathrm{BS}$ in the selected ranging slot. If no other SS has transmitted the same code in the same slot, the code will be successfully received by the BS. As the BS cannot identify the SS that has sent the CDMA ranging request, upon successfully receiving a CDMA ranging code, the BS broadcasts a ranging response (CDMA_Allocation_IE) message that advertises the received ranging code as well as the ranging slot (i.e., OFDM symbol number, subchannel, etc.) where the CDMA ranging code has been received. This information enables the SS that has successfully sent the ranging code to identify itself. The CDMA_Allocation_IE message contains all the needed transmission adjustments (e.g., time, power and possibly frequency corrections) that the SS should perform, and a status notification. The CDMA_Allocation_IE message also allocates uplink channel resource for the successful SS to transmit its bandwidth request information to the BS. In the unlikely event that the BS successfully captures a code even if two or more SS transmits the same code in the same slot, those SSs will transmit their bandwidth requests in the same allocated uplink channel resulting in a collision. In this case, all the SSs involved in the collision can repeat the bandwidth request procedure using a backoff algorithm [1], [2]. Due to the rare occurrence of this event, we do not consider the possibility of code capturing in this paper.

Upon receiving a CDMA_Allocation_IE message with a "continue" status, the SS waits for expiration of its periodic ranging timer before continuing the ranging process in the same manner as the initial ranging, but with a ranging code randomly chosen from the subset of codes allocated for periodic ranging. In the OFDMA ranging mechanism, the periodic ranging timer is controlled by the SS rather than the BS. After a contention transmission for ranging or bandwidth request, the SS waits for a Data Grant Burst Type IE in a subsequent map (or waits for a RNG-RSP message for initial ranging). Once received, the contention resolution is complete. The SS considers the bandwidth request lost if no data grant has been given within timeout T16 (or no response within timeout T3 for initial ranging) as shown in Fig. 2. The SS shall then increase its backoff window by a factor of two, as long as it is less than the maximum backoff window. The SS shall randomly select a number within its new backoff window and repeat the deferring process described above. 


\section{$3 \quad$ Performance Models}

To enable theoretical analysis of the performance of request transmissions over the OC-ALOHA ranging channel, we present a CTMC M/M/1 model initially developed in [6] that approximates the DTMC model [11]. This section also considers how measures such as number of backlogged users, arrival rate and the critical state estimation can be utilized for system stability. To provide this stability, we apply our model to derive two algorithms respectively for cases in which the number of code slots in the ranging channel per frame is fixed or can be dynamically readjusted.

\subsection{Assumptions}

As shown in Fig. 1, a ranging channel consists of two sub-channels and several symbol slots or ranging slots in the OFDMA uplink MAC frame, each divided into a number of ranging codes or ranging code slots. Each code slot accommodates transmission of a CDMA ranging code selected from the subset of codes specified for the intended ranging operation.

As aforementioned, we consider CDMA ranging code requests such as initial ranging, periodic ranging and bandwidth requests. Assume first that the total number of requests generated by all SSs behaves according to a Poisson process with rate $\lambda$, such that the probability of $k$ requests being generated per frame amounts to

$$
p[k]=\frac{e^{-\lambda} \lambda^{k}}{k !}, k=0,1,2, \ldots
$$

where $\lambda$ is the average number of ranging requests per frame. This is a reasonable assumption as the SSs generally act independently of each other.

If two or more ranging request codes are sent by different SSs using the same ranging code in the same ranging slot in a OFDMA MAC frame, then there will be a collision and the BS will obtain no information about the sources of the requests. If one SS sends a ranging code request in a given ranging slot, the ranging request is correctly received.

To simplify the evaluations of the behavior of the ranging sub-channel, we assume immediate feedback over the downlink channel. This allows the use of a single Markov chain rather than a number of independent Markov chains in the analysis. As this work focuses on the performance of the MAC layer, we assume that the physical transmission is perfectly received if it does not encounter a collision. More specifically, this assumes that the multiple access interference (MAI) between CDMA codes, does not result in transmission errors.

\subsection{Mean Delay Time and First Exit Time Analysis}

In order to find the access delay of requests in the ranging channel, we define $X_{i}$ as the number of backlogged users at the end of the $i^{t h}$ frame. Then sequence of $X_{i}$ 's forms a Markov chain. As noted in [12], the analysis encounters numerical problem as the number of time slots (corresponding to the code sots in this paper) per frame for packet transmissions increases. Here the number of 
code slots $(n)$ is defined as the product of the number of codes and the number of slots available in one frame. As the number of code slots $(n)$ increases, the number of successful requests per frame asymptotically approaches a Poisson distribution with mean $k e^{-k / n}$ where $k$ is the number of requests attempting random access transmission each choosing one of $n$ the available code-slots. Under this condition of "large" $n$, if we further approximate the model by ignoring the discrete nature of the framed structure, we can analyze the system by a continuous time Markov chain. Consider that the time for successful transmission and inter-arrival times for code requests are exponentially distributed with mean $1 / \mu_{k}$ and $1 / \lambda_{k}$, respectively, where is the total number of backlogged users and newly arrived requests. Then the state of the OC-ALOHA channel can be approximated [6] by a generalized $\mathrm{M} / \mathrm{M} / 1$ queuing model with arrival rate $\lambda_{k}=\lambda$ and service rate $\mu_{k}=k e^{-k / n}$. Under $\mathrm{M} / \mathrm{M} / 1$ formulation, we can obtain $p[$ the number of packets in systems $=k]$ as:

$$
\begin{gathered}
p_{k}=\frac{\lambda_{k-1}}{\mu_{k}} p_{k-1}=p_{0}\left(\prod_{i=1}^{k} \frac{\lambda_{i-1}}{\mu_{i}}\right) \\
p_{0}=\left[1+\sum_{k=1}^{\infty} \prod_{i=1}^{k} \frac{\lambda_{i}}{\mu_{i}}\right]^{-1}
\end{gathered}
$$

The mean delay time (MDT), $\bar{D}$ is given by:

$$
\bar{D}=\left(\frac{1}{\lambda} \sum_{k=1}^{\infty} k p_{k}\right)-\frac{1}{2}
$$

where $1 / 2$ accounts for the average waiting frame time for new bandwidth requests due to frame synchronization which was not included in the discrete time models.

We consider next the FET. As defined in [12], FET is the average time for the system to make a first exit into the unstable region starting from an initially empty system, which means that all code slots in the frame are empty. In other words, the stability definition is as follows: a ranging channel is said to be stable if its service rate $\mu_{k}$ is greater than $\lambda$. Otherwise, the channel is said to be unstable.

Define $k_{c r}$ to be the critical state of channel, as the minimum of $k$ at which $\mu_{k}<\lambda$.

$$
k_{c r}=\underset{\mu_{k}<\lambda}{\arg \min } k
$$

Let the transition probabilities from state $i$ to $i+1$ and from $i$ state to $i-1$ be $\theta_{i}=\frac{\lambda_{i}}{\lambda_{i}+\mu_{i}}$ and $\bar{\theta}_{i}=1-\theta$, respectively. Then the mean transition time $t_{i, i+1}$ from state $i$ to $i+1$ can be written as:

$$
t_{i, i+1}=\frac{1}{\theta_{i}}\left[\frac{\theta_{i}}{\lambda}+\bar{\theta}_{i}\left(\frac{1}{\mu_{i}}+t_{i-1, i}\right)\right]
$$

where $t_{01}=\frac{1}{\lambda}$. Using (6), we can express the FET $t_{0, k_{c r}}$ as: 


$$
t_{0, k_{c r}}=\sum_{i=0}^{k_{c r}-1} t_{i, i+1}
$$

\subsection{System Stability Evaluation}

With the stability measure previously defined in Subsection 3.2, we demonstrate how the system can be evaluated by estimating the arrival rate and number of backlogged users to determine whether the stable state can be consistently maintained. This is achieved by estimating the critical state of the system, beyond which the system will go into the unstable state. We can obtain the estimated arrival rate using the first order autoregressive model as follows:

$$
\tilde{\lambda}(i)=\theta_{\lambda}(i)+\left(1-\theta_{\lambda}\right) \tilde{\lambda}(i-1)
$$

where $n s(i), \tilde{\lambda}(i)$ are the number of successes and the estimated arrival rate in the $i^{\text {th }}$ frame, respectively, and $\theta_{\lambda}$ is the scalar smoothing factor in the arrival rate estimator (assuming $0 \leq \theta_{\lambda} \leq 1$ ).

Substituting $\tilde{\lambda}(i)$ into (5)-(7), we then obtain the estimated critical state, $\tilde{k}_{c r}$ and the estimated FET at the $i^{\text {th }}$ frame, $\hat{F}(i)$ as follows:

$$
\begin{gathered}
\tilde{k}_{c r}=\underset{\mu_{k}<\tilde{\lambda}}{\arg \min } k \\
\hat{F}(i)=t_{0, \tilde{k}_{c r}}=\sum_{i=0}^{\tilde{k}_{c r}-1} t_{i, i-1}
\end{gathered}
$$

In addition, the number of backlogged users can be estimated through the average number of access attempts until the ranging request is successful. When a SS sends a ranging channel transmission such as a bandwidth request to the BS using a randomly selected request code and the BS does not return any information to the SS, a code collision has occurred. Therefore, we assume that the SS reports the number of access attempts to the BS until the ranging request is successful. Thus, to estimate the number of backlogged users, we define the average number of access attempts until the ranging request is successful, $c(i)$ given by

$$
c(i)=\frac{1}{n s(i)} \sum_{k=1}^{n s(i)} t x(k)
$$

where $n s(i)$ and $t x(k)$ are the number of successful requests in the $i_{t h}$ frame and the number of transmission attempts of the $k^{t h}$ users, respectively.

Applying (9), we can estimate the number of backlogged users, $\tilde{b}(i)$, as:

$$
\tilde{b}(i)=\theta_{b} c(i)+\left(1-\theta_{b}\right) \tilde{b}(1-i)
$$

where $\theta_{b}$ is a scalar smoothing factor in the estimator (assuming $0 \leq \theta_{b} \leq 1$ ).

Based on the estimated arrival rate $(\tilde{\lambda}(i))$, the estimated critical state $\left(\hat{k}_{c r}\right)$ in $\hat{F}(i)$ and the estimated number of backlogged users $(\hat{b}(i))$ from $(8)-(12)$, we develop two novel algorithms for system stability. 


\section{Algorithm 1: Estimation-based Stabilization (ES) Algorithm with the Fixed Number of Ranging Code-Slots}

1. At frame $i=0$, set the number of code slots $(n)$ for contention;

2. Estimate $\tilde{\lambda}(i), \tilde{k}_{c r}$ in $\hat{F}(i)$ and $\hat{b}(i)$, in each ranging sub-frame using (8)-(12);

3 . Each SS that has a ranging request to transmit obtains permission to transmit with probability $\gamma(i)$ and selects a code slot at random:

$$
\gamma(i)=\tilde{k}_{c r} /\left(\hat{b}(i)+\tilde{k}_{c r}\right),(0 \leq \gamma(i) \leq 1)
$$

4. Go to step (2) for time frame $i+1$.

This algorithm estimates the permission probability $(\gamma(i))$ for transmitting ranging requests in frame $i+1$ for the situation where a fixed number of code slots are available in the contention-based ranging channel per frame. System stability is maintained by controlling the permission probability $\gamma(\mathrm{i})$.

\section{Algorithm 2: Estimation-based Stabilization (ES) Algorithm with Dynamic Ranging Code Allocation}

$\delta(1)=0$

$n_{\text {min }}$ : the initiated number of code slots or the minimum number of code slots;

1. At frame $i=1$, set the minimum number of code slots $\left(n_{\text {min }}\right)$ for contention;

2. Estimate $\tilde{\lambda}(i), \tilde{k}_{c r}$ in $\hat{F}(i)$ and $\hat{b}(i)$ in a ranging sub-frame $i$ using(8)-(12) with

$$
I_{n}(i)=n_{\min }+\delta(i)
$$

where $I_{n}(i)$ is the number of code slots which would dynamically be allocated by the variation parameter $(\delta(i))$ on the basis of $n_{\text {min }}$.

3. Calculate the permission probability $(\gamma(i))$ in this frame and the variation parameter $(\delta(i+1))$ for the next frame.

$$
\begin{aligned}
& \gamma(i)=\tilde{k}_{c r} /\left(\hat{b}(i)+\tilde{k}_{c r}\right) ; \quad(0 \leq \gamma(i) \leq 1) \\
& \delta(i+1)=I_{n}(i)-\left\lceil I_{n}(i) *(\gamma(i))\right\rceil
\end{aligned}
$$

4. Go to step (2) for time frame $i+1$.

This algorithm adjusts dynamically the number of code slots for ranging channel per frame based on the minimum number of code slots $\left(n_{\text {min }}\right)$. After estimating $\tilde{k}_{c r}$ in $\hat{F}(i)$ and $b(i)$ in frame $i$, the BS calculates the permission probability $(\gamma(i))$ that indicates the degree of the instability of the system. The variation parameter $(\delta(i+1))$ is kept small if $\gamma(i)$ is close to one indicating a stable system; it is increased as $\gamma(i)$ gets smaller. 


\section{$4 \quad$ Numerical and Simulation Results}

In this section, we present numerical and simulation results obtained using MATLAB. We assume that the arrival process of access requests at each SS is Poisson with rate $\lambda$, and let the number of backlogged requests vary according to the specified protocol. The duration of each simulation run is 1000 frames. Other basic assumptions are the same as given in Section 3.

In [6], we observed that FET increases with the number of code-slots, $n$, and decreases as normalized arrival rate increases. This is because the number of collisions increases when $n$ is reduced or the arrival rate is increased, resulting in a decrease in FET. Figs. 3(a) and (b) illustrate the trading relations among the estimated arrival rate, the estimated critical state, and the estimated number of backlogged users given by (8)-(10). The results have been obtained with $n=21$, $\theta_{\lambda}=0.05, \theta_{b}=0.8$, and the arrival rate is increased from 3 to 7 at around the $340^{t h}$ frame, and subsequently decreased to 4 at around the $670^{\text {th }}$ frame. Fig. 3(b) shows that the estimated arrival rate quickly decreases to 0 at around the $650^{\text {th }}$ frame. This is indicated by the point in Fig. 3(a) where the estimated number of backlogged users exceeds the estimated critical state as shown in around the $440^{t h}$, the $525^{t h}$ and the $610^{t h}$ frames, which implies that the system has a high probability of falling into the unstable state. In this case, system stability can be maintained by implementing measures to reduce collisions, such as using a contention resolution algorithm or a mechanism to dynamically adjust the number of code-slots in the ranging sub-channel.
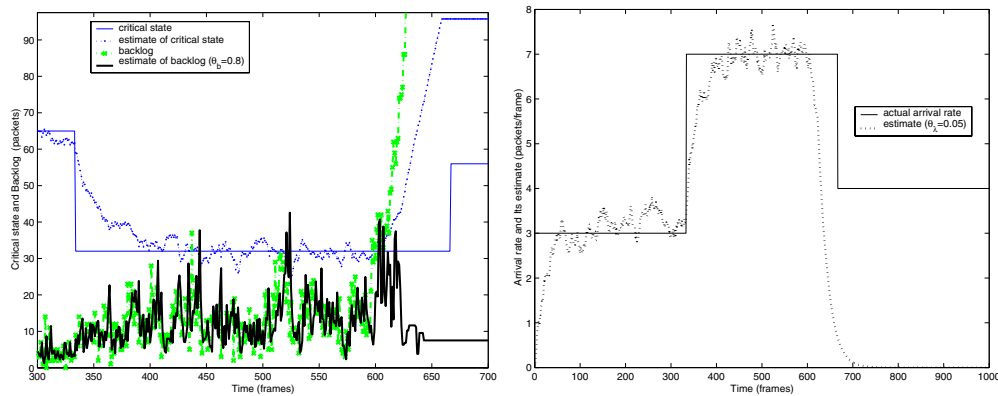

(a)Critical state and backlogged users (b)Actual arrival rate and its estimate

Fig. 3. Example of system stability evaluation

Figs. 4 and 5 illustrate the stability performance of algorithm 1 and 2, respectively, as presented in section 3. The results in Fig. 4 have been obtained using the following parameters for simulations: $n=90, \theta_{\lambda}=0.05, \theta_{b}=0.8$, the actual arrival rate is increased from 15 to 33 , and subsequently decreased to 20 . In Fig. 4(a), we can clearly observe that algorithm 1 always maintains the stable interval between the critical state and the number of backlogged users by 

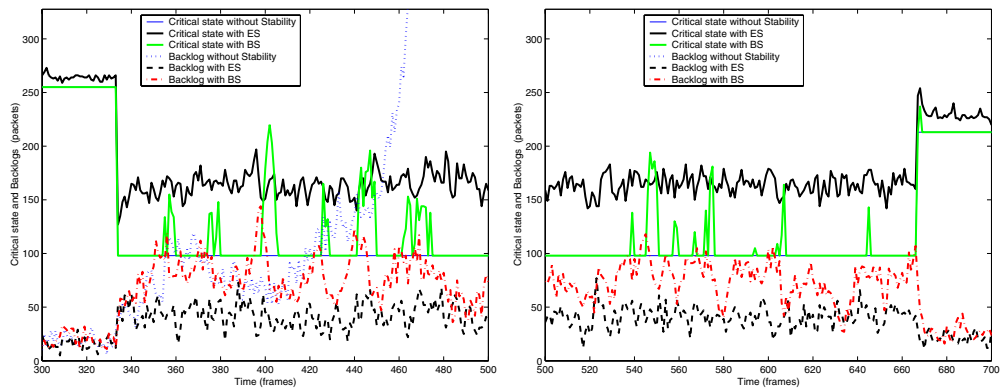

(a)Critical state and backlogged users

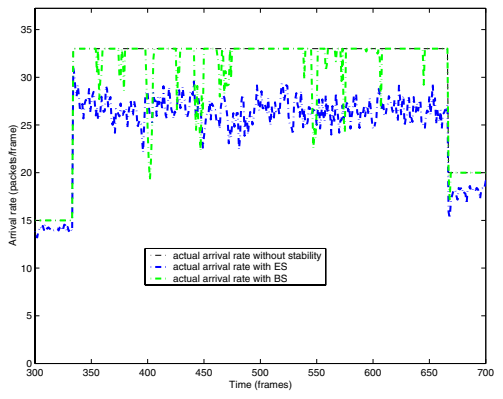

(b)Arrival rate

Fig. 4. Comparisons of performance of OC-ALOHA channel with a fixed number of code slots with (1) Estimation-based Stabilization (ES) Algorithm 1, (2) PseudoBayesian Broadcasting Stabilization (BBS) and (3) no stabilization: (a) Critical state and backlogged users; (b) Arrival rate

strictly restricting the arrival rate (at the channel) even if the actual arrival rate (at the SSs) is quickly increased from 15 to 30 at around the $335^{\text {th }}$ frame as shown in Fig 4(b). By doing so, the numbers of backlog users at the same frame is increased from 20 to 60 while the critical state is decreased 260 to 125 , and then they keep almost identical values with a little fluctuation when the frame proceeds until around the $665^{\text {th }}$ frame. On the contrary, the results for the Pseudo-Bayesian algorithm in Fig. 4 (b) show that the number of backlog users sometimes exceeds the critical state between the 335 th frame and the $665^{\text {th }}$ when the arrival rate is high. Specifically, the number of backlogged users exceeds the critical state at around the $355^{t h}$, the $395^{t h}$, the $440^{t h}$, the $545^{t h}$, and the $605^{\text {th }}$ frames and then this unstable state is stabilized gradually at the following frames. These results show that our estimation-based stabilizing algorithm derived from the CTMC M/M/1 model using FET and number of backlogged users as the stability measures achieves better stabilization than the Pseudo-Bayesian Broadcasting algorithm. 


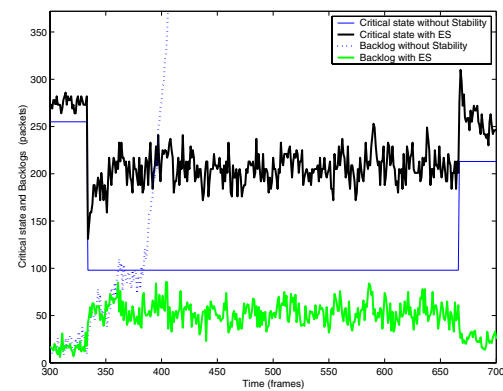

(a)Critical state and backlogged users

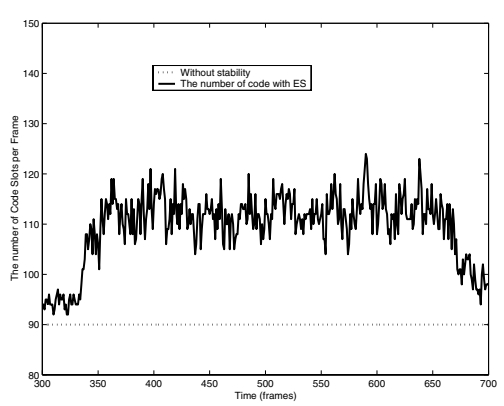

(b)The number of code slots per frame

Fig. 5. Performance of Algorithm 2 with the dynamic adjustment of number of code slots

To evaluate algorithm 2, we use the same simulation parameters as before, except that the number of code slots per frame is varied by estimating FET and the number of backlogged users. Figs. 5(a) shows that the system stability is always maintained even in the time interval that the system may fall into the unstable state as indicated by Fig. 4(b). Based on algorithm 2, the number of code slots per frame is adjusted upward from around 95 up to 120 and then downward to 100 when the actual arrival rate is increased from 15 to 33 and then decreased to 20 .

In practice, the performance of our algorithms depends on the error performance of the physical layer given the particular spreading codes selected by the SS and the distribution of the power levels. Therefore, the evaluations can be extended to incorporate physical layer models. We leave these for future work.

\section{Conclusions}

In this paper, we have presented a new analytical technique based on the CTMC $\mathrm{M} / \mathrm{M} / 1$ model for the contention-based CDMA ranging channel in IEEE 802.16a/d WMANs. This OC-ALOHA channel is inherently unstable. The model enables system measures such as the arrival rate, system backlog and critical to be easily estimated and utilized for system stabilization. We have proposed two novel stabilization algorithms, respectively for cases that the number of ranging code per frame is fixed or can be dynamically readjusted. In the former case, we have shown that system stability is always maintained by strictly restricting the ranging requests with permission probability $(\gamma(i))$. In the latter case, we have shown that the algorithm always maintains channel stability by dynamically allocating the number of code slots with permission probability $(\gamma(i))$ and the variation parameter $(\delta(i))$. Our novel approach of using a CTMC M/M/1 model to study system stability and to derive stabilization algorithms can be applied to other random access protocols and can be extended by incorporating more realistic physical layer models. 


\section{Acknowledgments}

This work was supported by the Post-doctoral Fellowship Program of Korea Science \& Engineering Foundation (KOSEF) and the Natural Sciences \& Engineering Research Council of Canada through grant RGPIN 44286-04.

\section{References}

1. IEEE Standard for Local and Metropolitan Area Networks, "Part 16: Air Interface for Fixed Broad Wireless Access Systems - Amendment 2: Medium Access Control Modification and Additional Physical Layer Specification for 2-11GHz", IEEE Std 802.16aTM-2003.

2. Draft IEEE Standard for Local and metropolitan area networks, "Part 16: Air Interface for Broadband Wireless Access Systems", IEEE P802.16-REVd/D5-2004, May 2004.

3. http://www.wimaxforum.org/news/downloads/WiMAXWhitepaper.pdf

4. A. Brand and H. Aghavami, Multiple Access Protocols for Mobile Communications-GPRS,UMTS and Beyond, Wiley, 2002.

5. S. Simoens, P. Pellati, J. Gosteau and K. Gosse, "The Evolution of 5GHz WLAN toward Higher Throughputs", IEEE Wireless Communications, vol. 10, pp. 6-13, December 2003.

6. J.-J. Won, H.-H. Seo, C.-H. Cho, H.-W. Lee and V.C.M Leung, "Peformance Analysis of Contention-based Multiple Access Protocol in IEEE 802.16a Wireless MAN", Proc. 9th CDMA International Conference, pp. 334-338, Seoul, Korea, Oct. 2004.

7. S. Mangold, S. Choi, G. R. Hiertz, O. Klein, and B. Walke, "Analysis of IEEE 802.11e for QoS Support in Wireless LANs", IEEE Wireless Communications, vol. 10, pp. 40-50, Dec. 2003.

8. G.-H. Hwang and D.-H. Cho, "Adaptive random channel allocation scheme in HIPERLAN type 2", IEEE Communications Letters, vol. 6, pp. 40-42, Jan. 2002.

9. G. Anastasi and L. Lenzini, "HIPERLAN/1 MAC Protocol: Stability and Performance Analysis", IEEE J. Selected Areas in Commun., vol. 18, pp. 1787-1798, Sept. 2000.

10. D. Bertsekas and R. Gallger, Data Networks, Prentice Hall, 1992.

11. W. Feller, An Introduction to Probability Theory and Its applications, vol. 1, Cornell University, Jan. 1950.

12. L. Kleinrock and S. S. Lam, "Packet Switching in a Multiaccess Broadcast Channel: Performance Evaluation", IEEE Trans. Commun., vol. Com-23, pp. 410-423, Apr. 1975.

13. L. Kleinrock, Queuing Systems, vol. 1: Theory, Wiley-Interscience. 